\title{
NECESIDADES EMERGENTES Y RESPONSABILIDAD SOCIAL UNIVERSITARIA
}

\author{
NATIVIDAD DE LA RED VEGA
}

Departamento de Sociología y Trabajo Social. Facultad de Educación y Trabajo Social. Universidad de Valladolid.

\section{RESUMEN}

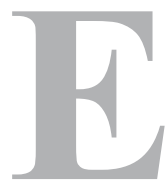

1 escenario social actual plantea nuevas dimensiones para un progreso humano que conlleva la promoción no únicamente de los recursos económicos, también de los recursos humanos y sociales que den cumplida respuesta a las necesidades sociales emergentes. Ello comporta nuevas exigencias de responsabilidad social que han de ser asumidas por personas, profesionales y organizaciones. La educación, y por tanto la universidad, tiene un papel clave en los procesos de responsabilidad social, que se ha de poner de manifiesto a través del ejercicio de sus funciones: la gestión, la docencia, la investigación y el compromiso social, en el contexto del que forma parte. Asumir estas funciones, desde la responsabilidad social, supone un compromiso de toda la organización universitaria con el desarrollo ciudadano, democrático y sostenible, y también, con la inclusión social y la riqueza de las redes sociales; en definitiva, con la calidad de vida.

Palabras clave: necesidades emergentes, organización, universidad, responsabilidad social, agentes, procesos.

\begin{abstract}
The current social climate raises new issues for human progress that involves not only economic resources but also the development of human and social resources to respond to emerging social needs. This creates new social responsibility requirements for people, professionals and organisations. Education and universities play a vital role in the processes of social responsibility, in terms of their management, teaching, research and social commitment within their own context. Assuming these functions from a position of social responsibility requires the commitment of the whole university, with democratic and sustainable citizenship, social inclusion and enriched social networks with a view to increased quality of life.
\end{abstract}

Key words: emerging needs, organisation, university, social responsibility, actors, processes. 


\section{INTRODUCCIÓN}

La globalización económica, técnica y de la información son ya un hecho incuestionable por su evidente realidad. Ante el avance de estos fenómenos globales, parece que encontramos desorientaciones en la globalización de la humanidad, de la ética, de la dimensión humana. En esta situación, no faltan quienes afirman que la globalización y la sociedad del conocimiento pueden significar la gran oportunidad para la humanidad. Para ello, se habrá de superar, en este mundo globalizado, una dinámica de progreso que ha pasado de ser un medio a ser un fin; cuando el progreso es el objetivo de la humanidad y deja de ser la humanidad el objetivo del progreso, se produce mucha muerte. La historia nos lo viene demostrando.

La humanización del progreso, el rostro humano de la globalización y de la sociedad del conocimiento, comporta el logro equilibrado del desarrollo en armonía con la ética global y con la solidaridad, para no solamente competir para ser más competitivos, sino también compartir siendo solidarios.

Como afirmó el premio Nobel de la Paz, Nelson Mandela, el reto del siglo debe ser el de globalizar la solidaridad y, por tanto, también la responsabilidad. Ello nos sitúa, por una parte, en el ámbito de los recursos no monetarios como el otro pie de camino de la globalización económico-monetaria y, por otra parte, ante la relación entre progreso y humanidad desde las nuevas bases de la moral.

Las dimensiones esenciales de la calidad de vida en nuestra realidad no están solamente vinculadas a lo económico y a lo monetario; así se constata ante situaciones afectadas por el desempleo, la soledad, la incomunicación, la crisis del medio ambiente, las vinculadas a la prolongación de la esperanza de vida, la inmigración, las revoluciones de tiempos, los desequilibrios norte-sur, la necesidad de repensar los modos y formas de cooperación internacional... Las múltiples dimensiones de solución a estos fenómenos están vinculadas a los recursos económicos, pero también a expresiones de cooperación, de riqueza social y capacidad creativa, también, a veces, no ausentes de confusión. Y ello porque incluso la asignación de los mismos recursos económicos ha de estar precedida de esas expresiones, y sólo desde la sensibilidad necesaria se contemplará el enfoque correcto de los respectivos presupuestos de diversas organizaciones públicas o privadas. Porque únicamente desde ese tipo de preparación y desde la gestión eficaz se aplicarán esos recursos a los fines que incidan en la calidad de vida de los destinatarios.

Aparece con cierta evidencia que hoy las leyes, los reglamentos, las normas, la racionalidad económica, la organización y la gestión, con ser necesarias, no son suficientes para garantizar la prosperidad moderna, el bienestar y el progreso con rostro humano.

Además, en este contexto de globalización, de nuevos conceptos de espacio y tiempo, y de inversión de medios-fines, han evolucionado las bases de la moral; hoy las bases de la moral están en la dignidad. ¿La dignidad de quién? En un mundo globalizado, la dignidad de todos, porque todos somos ya próximos; y la dignidad de cada uno, de cada persona, de cada organización, de cada institución, en definitiva, de la sociedad en la que vivimos.

\section{NECESIDADES Y PROBLEMAS. RECURSOS A POTENCIAR Y RESPONSABI- LIDAD SOCIAL DE LAS ORGANIZACIONES}

Surgen actualmente nuevas necesidades, y no tan nuevas, que interfieren en la calidad de vida de la ciudadanía y de la sociedad; necesidades cuya respuesta y satisfacción requiere de recursos económicos, pero también necesidades relacionadas con la inclusión y cohesión social, con los derechos cívicos y medioambientales, con la participación y la autodeterminación. Todo ello se produce en un escenario complejo y no ausente de crisis político-social que incide en la pérdida de confianza de la ciudadanía, en la credibilidad de 
las instituciones de representación política, como los partidos políticos o los gobiernos, en la incertidumbre de las organizaciones sociales como sindicatos y asociaciones, o de los programas que no responden fielmente a las dimensiones actuales de las necesidades.

Parece aún actual la expresión de Martínez cuando nos dice, hace ya más de 14 años, que "el Estado se ve cada vez más inhabilitado para satisfacer necesidades colectivas, las instituciones no muestran una capacidad de respuesta suficiente para adecuarse al nuevo escenario y las estructuras de representación confrontan desafíos cuya complejidad las supera, poniendo en evidencia en muchos casos su rigidez"1.

Todo ello incide en la nuevas formas que va adoptando la misma crisis del Estado de Bienestar, lo que se ha venido desarrollando al ritmo de varios factores, entre los que señala García Roca el debilitamiento de la cultura participativa y redistributiva, por lo que se hace necesario, para la solución de dicha crisis, en el contexto político, "ampliar los actores sociales en orden a crear y mantener los sistemas de protección" para fortalecer la participación social y ciudadana ${ }^{2}$.

En la búsqueda de respuestas a estas situaciones, se hacen necesarios también otros elementos del progreso humano, desde las nuevas bases de la moral, a partir de nuevas formas de confianza y de altruismo. Ello tiene su expresión en el desarrollo de nuevas dinámicas sociales, en nuevas realidades no económicas que se activan, por ejemplo, desde el descubrimiento de la importancia de dar y recibir y de la atención al tejido social basado en la reciprocidad y la responsabilidad colectiva. Se produce, así, en la sociedad, la riqueza social, que se concreta en lo que viene denominándose capital social ${ }^{3}$, porque genera el clima de confianza que aumente la eficiencia de cualquier grupo, organización o sociedad.

Sin entrar aquí en los debates sobre la idea de capital social, nos limitamos a recordar las aportaciones de diversos autores que se basan en el valor intrínseco y colectivo de las comunidades y las corrientes que surgen de estos grupos para apoyarse mutuamente. Los beneficios que se derivan de ese apoyo mutuo organizado, según algunos de los primeros planteamientos de diversos autores, van desde el "potenciamiento de la democracia", como señala Bourdie (1986), pasando por la "energía social" que nos describe Hirschan (1986), los "valores compartidos" que resalta Coleman (1988), la "riqueza cívica de los valores recíprocos y la cooperación" que nos muestra Putman (1993), la "importancia de la confianza y la cooperación para el progreso" que apunta Fukuyama (1995) o la importancia de la "confianza y la capacidad asociativa" que resalta Kliksberg (2000).

En estos procesos, la educación es la principal vía de potenciación de este capital de personas y grupos y también es un factor fundamental en la presencia de profesionales socialmente responsables. En ello, las organizaciones tienen un papel clave.

1 MARTÍNEZ, R. (1995), "Redes sociales. Más allá del individualismo y del comunitarismo", en Dabas, E., y Najamanovich D. (comp), Redes. El lenguaje de los vínculos: Hacia la reconstrucción y el fortalecimiento de la sociedad civil, Buenos Aires: Paidós, 338.

2 GARCÍA ROCA, J. (2004), Políticas y programas de participación social, Madrid: Síntesis, 43.

3 Consideramos aquí la expresión 'capital social' en lo que tiene de riqueza de dinámicas emprendedoras, de interacciones sociales con repercusión en la riqueza del tejido social. Existen perspectivas diferentes sobre lo que se denomina capital social; es apoyado por algunos autores, pero no faltan quienes cuestionan el concepto y el contenido, como, por ejemplo, NAVARRO LÓPEZ, V., "Crítica del concepto de capital social”, Sistema. Revista de Ciencias Sociales, 172, 2003, 27-36. También Marrero A., "La teoría del capital social, una crítica en perspectiva latinoamericana", Revista Arxius, $<$ www.rae.edu.uy/fcs/soc/curriculum/Amarrero>, tomado el 8 de septiembre de 2009.

Otros autores, entre ellos, Harriss y De Renzio (1997), nos advierten de algunos límites de los contenidos y posibles efectos del capital social. Además, señalan que la expresión 'capital social' es imprecisa y da lugar a malentendidos, pues se utiliza en referencia a contenidos bastante diferentes (vínculos familiares, organizaciones sociales, relaciones entre sociedad civil y Estado, marco político e institucional, normas sociales, etc.). Por otra parte, según cuál sea el concepto de capital social del que se parta, los proyectos orientados a incrementarlo pueden contribuir a reducir las desigualdades estructurales en las relaciones de poder, pero, a veces, también a incrementarlas. 
Porque desde las organizaciones se promueven los recursos humanos y sociales; también las normas y las pautas de conducta que generan la confianza y la cooperación entre la gente y en la sociedad en general, que tienen su expresión en la responsabilidad social. Por ello, la responsabilidad social se ha identificado como factor importante del progreso democrático y del desarrollo social y económico -por ejemplo, Coleman (1990), Putnam (1993) - y, sobre todo, como clave en el proceso de cambio social capaz de superar los problemas actuales, que nos indica Kliksberg (2005), relacionados con: la creciente brecha social a partir de la desigualdad en la distribución de ingresos, la riqueza y los recursos educativos; la crisis de participación y representación y responsabilidad política por la realización de los derechos sociales; la repercusión de nuestro desarrollo en el medio ambiente, el marcado retroceso de sentido, de valores y de aspectos ético-morales con repercusión en la gestión y el desarrollo humano.

La respuesta a las exigencias sociales actuales es competencia de toda organización, sea pública, privada o social. Competencia que habrá de estar además presente en las interacciones entre el Estado, el Mercado y la Sociedad, desde la ausencia de prejuicios y la visión de responsabilidad compartida. Es una expresión de la consciencia de la repercusión y del impacto social que tiene cualquier decisión que adopte cada uno de los agentes como organización, en lo social, en lo económico, en lo relacional.

Ello parece tener su expresión más evidente en el creciente uso que está teniendo la dimensión social: mercado social, inversión social, balance social, contabilidad social, capital social. Una importancia que será efectiva si evita algunas contraindicaciones, tal como nos señala Guerra Sotill: "Existe el riesgo de banalizar lo social y convertirlo en mera etiqueta limpiadora de conciencias, simple disfraz de una motivación básica y primariamente económica. En todo caso, lo social se incorpora, cada vez con más fuerza, como dimensión que complementa a lo económico-financiero, como criterio de gestión y evaluación de resultados, en un equilibrio dificil y esquivo, pero necesario"”.

La mayor exigencia de lo social se hace más necesaria ante las múltiples dimensiones de la problemática actual, lo que exige diversas perspectivas de abordaje desde la responsabilidad de cada uno y desde la responsabilidad de la organización como un todo. Podemos, pues, considerar que una organización socialmente responsable ha de equilibrar y responder de sus impactos, sean éstos económicos, sociales y medioambientales o relacionados directamente con el fin específico que la caracteriza (educación, salud, servicios sociales...). Todo ello tiene unos efectos ineludibles de responsabilidad social también en los enfoques estratégicos y de gestión, lo que comporta, en las situaciones complejas actuales, la presencia de sistemas integrados de calidad, rindiendo cuentas, mediante auditorías externas, para evitar que la responsabilidad social no se quede en algo estético, en meras declaraciones de intenciones. Igualmente, se trata de generar procesos y resultados que estén alineados con las actitudes y acciones responsables de todos los miembros de la organización. De ahí que la responsabilidad social organizacional va más allá del compromiso ético y responsabilidad social individual o profesional, construyendo y aplicando, en el día a día, la responsabilidad social de toda la organización, desde el enfoque de la complejidad, la interdependencia generada entre el todo (la organización) y las partes (el personal y el resto de los grupos de interés) ${ }^{5}$. Estos aspectos precisan, además, del concurso de los Estados para impulsar políticas públicas que salvaguarden los asuntos de interés social.

4 GUERRASOTILLO A. (2005), "Sobre responsabilidad social empresarial", en <www.analitica.com/va/sociedad/artículo/5900497.asp>, tomado el 17 de agosto de 2009.

5 BARRANCO, C., y RED VEGA, N. (2008), "Responsabilidad social organizacional y trabajo social", Ponencia presentada en el Congreso Internacional Interdisciplinar celebrado en la Universidad de Santiago el día 3 de septiembre de 2008 (en prensa). 
Parafraseando a Maturana, generar la responsabilidad social organizacional es exponerse la organización a sí misma y las personas vinculadas a la misma en sus preferencias y selecciones y hacerse cargo de las posibles consecuencias que pueda tener sobre los otros, lo que la organización y cada persona hacen ${ }^{6}$. Las consecuencias de toda organización van más allá de las dimensiones específicas de la misma y tienen una repercusión en la globalidad que nos envuelve, pues todo lo que sucede en cualquier parte está integrado en lo que E. Morin denomina conciencia y ciudadanía planetaria ${ }^{7}$.

\section{RESPONSABILIDAD SOCIAL UNIVERSITARIA}

La universidad, como organización, es un escenario privilegiado para la potenciación de recursos humanos y sociales y para la promoción de profesionales socialmente responsables en un mundo con nuevas necesidades. Por tanto, desde una gestión responsable, se ha de implicar en una formación que responda a dichas necesidades, a través de la docencia, la investigación, la vinculación al medio y la gestión universitaria.

Entre los desafíos universitarios más importantes, actualmente encontramos el de influir en los acontecimientos sociales, sin despreocuparse de su incidencia en los procesos sociales con identidad regional sostenibles que repercutan en la potenciación de bienestar de la sociedad de la que forma parte.

Así, la universidad se legitima socialmente en la medida que, desde la adecuada capacidad de análisis del contexto y la relación fluida y clara con los grupos y realidades sociales, responde a la promoción y calidad de vida del entorno del que forma parte. Ello requiere una cuidada sensibilidad hacia las necesidades y demandas sociales y que esta dimensión prevalezca siempre, en la docencia, la investigación, la gestión y las relaciones con la sociedad, sobre los intereses, las lógicas o los juegos de poder internos de la propia organización, más o menos corporativista, y ajenos a los de la sociedad en la que se sitúa.

Desde este enfoque, la responsabilidad social de las universidades es definida como "una política de calidad ética del desempeño de la comunidad universitaria (estudiantes, docentes y personal administrativo) a través de la gestión responsable de los impactos educativos, cognitivos, laborales y ambientales que la universidad genera, en un diálogo participativo con la sociedad para promover un desarrollo sostenible" (OEA-BID8). Ello plantea un desafío importante a las universidades actuales, esto es, su incidencia en las comunidades de aprendizaje mutuo para el desarrollo, a través de comportamientos socialmente responsables ante las necesidades y recursos sociales orientados al potenciamiento del progreso humano desde la propia universidad y con los stakeholders ${ }^{9}$. El desarrollo de las interacciones sociales se produce a través de los sentimientos, la confianza e información recíproca. Ello refuerza la cooperación y genera valor en las personas implicadas en

6 MATURANA, H. (2004), "Objetividad, responsabilidad y ética”, <http://www.periodismo.uchile.cl/noticias/2004/ anoacademicoinaug.html>, tomado el 25 de abril de 2006.

7 MORIN, E. (2001), Los siete saberes necesarios para la educación del futuro, Barcelona: Paidós. Puede verse también sobre este tema y de este mismo autor (1996), «El pensamiento ecologizado», <http://www.ugr.es/ pwlac/G12_01Edgar_Morin.html>, tomado el 25 de abril de 2008. MORIN, E. (2007), «Fronteras de lo político», <http://www.globalizacion.org/ciudadania/MorinFronteraPolitico.htm>, tomado el 25 de abril de 2008.

8 OEA-BID (2007), “Cómo enseñar Ética, capital social y Desarrollo en la Universidad? Estrategias de RSU”, apuntes del módulo 2: Responsabilidad Social Universitaria: ética desde la organización, p. 21.

9 En la definición de un stakeholder, influye actualmente la perspectiva que le otorga a dicho análisis el concepto de responsabilidad social que exige ubicar a los stakeholders en el centro o eje de la gestión de la organización, lo cual implica, como recuerda Adela Cortina (2006: 114), que "debe formar parte del núcleo duro de la empresa, de su gestión básica, no ser «algo más», no ser una especie de limosna añadida que convive tranquilamente con bajos salarios, mala calidad del producto, empleos precarios, incluso explotación y violación de derechos básicos. La buena reputación se gana con las buenas prácticas, no con un marketing social que funciona como maquillaje de un rostro poco presentable". 
estas dinámicas y en las personas que están en su perímetro y en el refuerzo del cambio social.

La UNESCO (1998) plantea, en la Declaración Mundial sobre la Educación Superior para el siglo XXI, en unos de los puntos relacionados con la responsabilidad social universitaria (artículo 6 de la declaración), la responsabilidad que tienen las universidades en las orientaciones de largo plazo que permitan resolver las necesidades y aspiraciones sociales, inculcando esta responsabilidad a los estudiantes. Igualmente, establece, en el mencionado artículo 6, letra (b): "La educación superior debe reforzar su servicio a la sociedad $y$ en especial sus actividades para eliminar la pobreza, la intolerancia, la violencia, el analfabetismo, el hambre, la degradación ambiental y la enfermedad, utilizando principalmente enfoques transdisciplinarios e interdisciplinarios en el análisis de los temas y los problemas".

Cuando la responsabilidad social en todo el ámbito universitario adquiere un lugar central en su visión, valores y misión, y no es algo accesorio, se produce un cambio significativo que afecta a toda la organización y a las relaciones con el contexto del que forma parte.

\section{DISTINTAS FORMAS DE ENTENDER LA UNIVERSIDAD}

\begin{tabular}{|l|l|}
\hline Centrada en contenidos. & $\begin{array}{l}\text { Centrada en contenidos, actitudes y valores. } \\
\text { Formación de ciudadanos competentes en sus fun- } \\
\text { Formación de profesionales productivos. }\end{array}$ \\
$\begin{array}{l}\text { Orientada a las necesidades del mercado de de } \\
\text { trabajo. }\end{array}$ & $\begin{array}{l}\text { Orientada a anticiparse a las necesidades de la so- } \\
\text { ciedad en su conjunto. }\end{array}$ \\
$\begin{array}{l}\text { Uso social basado en el estatus, enriquecimiento } \\
\text { y reconocimiento individual y en el crecimiento } \\
\text { económico. }\end{array}$ & $\begin{array}{l}\text { Uso social basado en la contribución al bien colecti- } \\
\text { vo, la construcción social y al desarrollo humano. }\end{array}$ \\
\hline
\end{tabular}

FUENTE: Propuesta de reporting de RSC en las universidades públicas: aplicación al caso de la UNED. (2008).

La Declaración de Talloires ${ }^{10}$, elaborada en 2005 por las 27 universidades firmantes de diversos continentes y países, recoge con claridad la forma de entender la responsabilidad social en una serie de compromisos entre los que destacamos aquí:

- Expandir nuestros programas de compromiso cívico y responsabilidad social fundamentados en principios éticos y a través de la enseñanza, la investigación y el servicio público.

- Asumir la responsabilidad pública mediante el ejemplo personal y las políticas y mejores prácticas de nuestras instituciones de educación superior.

- Construir un marco institucional para motivar, premiar y reconocer las buenas prácticas en el servicio social realizado por estudiantes, docentes, personal administrativo y en las comunidades afiliadas a programas de compromiso social de nuestras universidades.

- Asegurar que los parámetros de excelencia, el debate crítico, la investigación y el juicio crítico sean aplicados al compromiso comunitario, con la misma rigurosidad que se tiene en otras formas de la actividad universitaria.

- Elevar el interés en las agencias gubernamentales, las empresas, las organizaciones comunitarias y los organismos internacionales en relación a la contribución de la educación superior al avance social y el bienestar colectivo. Colaborar con otros sectores para

10 Equipo de rectores en Declaración sobre Funciones civicas y responsabilidad social de la educación superior, Talloires, Francia, septiembre de 2005. 
asegurar mayores impacto y ganancias sociales y económicas sostenidas para nuestras comunidades.

- Apoyar y promover asociaciones académicas, de carácter internacional, regional y nacional, como parte del esfuerzo de fortalecimiento del compromiso cívico universitario y del reconocimiento académico al servicio y acciones docentes y de investigación en el ámbito de la responsabilidad social universitaria.

- Participar activamente en actividades cívicas de importancia en nuestras comunidades.

\subsection{Responsabilidad social universitaria, principios y metodología}

El nuevo Espacio Europeo de Educación Superior establecido por el plan de Bolonia es una ocasión no únicamente para la homologación de créditos y de planes de estudios, es también una ocasión importante para recuperar y reforzar la misión de la universidad socialmente responsable desde espacios de participación de los stakeholders en los diferentes ámbitos del quehacer docente, investigador, de extensión y de gestión de las universidades ${ }^{11}$.

Ello comporta, como mínimo, mayor atención, por parte de las universidades, a los principios que fundamenten su responsabilidad universitaria y la dimensión del aprendizaje como servicio.

Tal como nos indica el profesor Rodríguez Fernández, cabe destacar como principios de la responsabilidad social universitaria ${ }^{12}$.

- Justicia, equidad, libertad de pensamiento, integridad y compromiso con la ciudadanía.

- Servicio público nacional e internacional.

- Incorporación de la responsabilidad social en el propio gobierno, organización, planificación y gestión de la universidad en todos sus niveles.

- Identificación objetiva de las partes interesadas.

- Organización, dirección y gestión participativas.

- Transparencia, evaluación de resultados desde una perspectiva múltiple y rendición de cuentas.

- Compromiso ético, interculturalidad, defensa de los derechos humanos, respeto de la diversidad, igualdad de oportunidades, preocupación por las generaciones futuras y sostenibilidad.

- Enfoque global y a largo plazo a favor del conjunto de la sociedad.

- Adopción de códigos formales de responsabilidad social.

Estos principios, asumidos por todos los órganos universitarios, facilitan la adecuación necesaria para afrontar los cambios ante situaciones de incertidumbre, conflictos culturales y divergencia de intereses.

11 En la última generación de los estatutos de las universidades aparece la preocupación por estas dimensiones, con frecuencia referidas a situaciones concretas. Así, por ejemplo, en la Universidad de Valladolid, en el artículo 3, se recoge que se asumen "como valores inspiradores de su actividad, la promoción de la paz y la cooperación entre los pueblos, la transformación social y la creación y difusión de formas culturales críticas y participativas, orientando el cumplimiento de sus fines bajo los principios de igualdad, libertad, justicia y solidaridad".

12 RODRÍGUEZ FERNÁNDEZ, J. M. (2009): "Responsabilidad social universitaria. Desafíos ante un cambio de época", en Actas de las Jornadas sobre Responsabilidad Social en la Universidad, organizadas por la Universidad de Valladolid, 28 y 29 de abril de 2009, pp. 9 y ss. 
Nos advierte de la Cuesta que, puesto que en la sociedad del conocimiento las relaciones entre los principales actores implicados han cambiado radicalmente, la universidad ha de analizar el nuevo entorno y afrontar con audacia los retos de futuro, desde una perspectiva de profundo compromiso con la función última que le es inherente: poner el conocimiento al servicio del ser humano, de su progreso ético, realización personal, desarrollo profesional y vinculación solidaria con los intereses generales ${ }^{13}$. De aquí, se deriva la importancia que está adquiriendo la metodología de lo que viene denominándose el aprendizaje-servicio.

Se trata de un sistema metodológico de aprendizaje que trata de generar beneficios en tres ámbitos de la educación superior: el currículum académico, la formación en valores y la vinculación con la comunidad. Esta metodología se planifica en función del proyecto educativo institucional, de manera integrada con los contenidos curriculares y sostiene simultáneamente una intención pedagógica (mejorar la calidad de los aprendizajes académicos, poniendo en práctica todos los conocimientos conceptuales, procedimentales y actitudinales adquiridos, y desarrollando nuevos aprendizajes y capacidades en la búsqueda de alternativas de desarrollo integral) y una intención solidaria (ofrecer una respuesta participativa a una necesidad social efectivamente sentida por la comunidad), consiguiendo efectos significativos, sólidos y duraderos tanto para los estudiantes como para la comunidad ${ }^{14}$.

Se trata, pues, de una responsabilidad en el aprendizaje de los estudiantes y también en la formación de futuros profesionales, vinculando ambos, aprendizaje y profesionalidad, con las necesidades sociales y contextuales. Por ello, hablamos de una Universidad capaz de asumir su responsabilidad ante las necesidades reales y las transformaciones necesarias a través de los fundamentos y conocimientos, y también a través de las prácticas necesarias.

Prácticas no solamente abordadas desde el enfoque técnico o no solamente económico o político: la complejidad de la realidades comporta mayor profundidad, que se plantea desde dimensiones éticas y estratégicas, no únicamente eficientes desde la perspectiva económica monetaria, también desde la dimensión de movilidad democrática de inclusión.

Tenemos, así, una Universidad que responde, como cualquier otra organización, a la "sed de ética" del mundo actual -en palabras de Kliksberg ${ }^{15}$ - y que se conduce a través de la responsabilidad social. Respuesta a la que no puede ser ajena ninguna organización. Para ello, como señala Morin ${ }^{16}$, se ha de armonizar la libertad de acción e iniciativa que caracteriza a toda organización en un mundo democrático con las preocupaciones por el mundo presente y futuro, sensible a la sociedad en la que se desarrolla.

\subsection{Responsabilidad social universitaria: algunas estrategias e indicadores}

Tal como venimos indicando, en la Universidad, podemos considerar cuatro estrategias generales de responsabilidad social universitaria, como nos indica Vallaeys ${ }^{17}$. En cada

13 DE LA CUESTA, M. (2009): "Modelo de responsabilidad social en las sociedades españolas", en actas de Jornadas sobre Responsabilidad social universitaria, organizadas por la Universidad de Valladolid, 28 y 29 de abril de 2009, pp. 13 y ss.

14 Cabe destacar las importantes aportaciones metodológicas y operativas sobre el aprendizaje-servicio de la profesora de la Universidad de Valladolid S. Lucas Mangas, con efectos muy destacados tanto para los alumnos como para el contexto en el que se desarrollan sus proyectos.

15 Véanse <http://www.iadb.org/ética>. <http://www.clarin.com/suplementos/libros/2006/03/10/masetica.pdf>, tomado el 20 de agosto de 2009.

$16<$ http://www.complexus.org/garcia/esppresapc.htm>, tomado el 10 de agosto de 2009 .

$17<\mathrm{http}: / /$ www.eumed.net/libros/2008b/402/Responsabilidad\%20Social\%20de\%201as\%20Universidades.htm>. 
una de esas estrategias se pueden considerar las respectivas acciones institucionales en torno a los indicadores correspondientes:

La gestión interna de la universidad es una estrategia de responsabilidad social que se pone de manifiesto cuando la universidad es una organización en la que se constata democracia, equidad (supresión de las segregaciones y corrección de los privilegios), transparencia (política y económica), haciendo de ella un modelo de desarrollo sostenible.

La responsabilidad social universitaria, nos indica de la Cuesta ${ }^{18}$, supone rendir cuentas a la sociedad de los avances positivos y negativos respecto a los compromisos asumidos con sus grupos de interés y, en general, en materia de derechos humanos, medio ambiente, buen gobierno y compromiso social. La RSU consiste en hacer bien las cosas para todos. Para ello, se debe utilizar la transparencia en la gestión, ya que ésta atrae mayores aportes de financiación y patrocinios y se crea una reputación y una buena imagen, mejoras de eficiencia en el uso de los recursos materiales (energéticos y otros), reducción de riesgos por conflictos con los grupos de interés (empleados, proveedores, etc.) y la necesidad de generar valores inmateriales (excelencia docente e investigadora, valores humanos...). Y esa gestión de calidad conllevará mejores resultados en el futuro.

Cabe considerar aquí, como ejemplos de indicadores, dentro de esta estrategia, los relacionados con:

a) La participación como: incorporación de stakeholders a procesos de planificación y evaluación, relación con los sindicatos y asociaciones estudiantiles, fomento de procesos democráticos internos, calidad total.

b) El medio ambiente: uso de sistemas de reciclaje en los recintos, uso de sistemas de ahorro de energía o energías alternativas, uso de tecnologías limpias o estudios de impacto ambiental de la gestión.

c) La inclusión social: igualdad de género en la designación de cargos de mayor responsabilidad, instalaciones y servicios para alumnos con discapacidad, apoyo a los alumnos en situación socio-económica especial, existencia de una cultura organizacional inclusiva.

d) El acceso a redes: empleabilidad de los titulados, capacidad emprendedora de los directivos, procesos de acreditación.

e) La toma de decisiones: diseño de políticas y procedimientos que consideren la participación de stakeholders, definición de lineamientos estratégicos, existencia de una filosofía de colaboración explícita.

En la universidad, la docencia constituye una estrategia clave para el aprendizaje socialmente responsable abierto a la comunidad y a las situaciones reales desde la articulación de la enseñanza y la investigación. De la Cuesta nos indica al respecto que "la educación superior, la investigación y la innovación no sólo son determinantes para afrontar los retos de una sociedad basada en el conocimiento, sino también para alcanzar una globalización solidaria y respetuosa de las identidades especificas, para garantizar el bienestar de los ciudadanos desde una perspectiva multidimensional, no sólo material, y para promover el desarrollo sostenible. En tal sentido, la Responsabilidad Social de las Universidades (RSU) es un medio para contribuir a todo ello" 19 .

Los indicadores correspondientes a este eje, como corresponde a la organización que nos ocupa, son claves y también cabe diferenciar los relacionados con:

18 DE LA CUESTA, M. (2009), "Modelos de responsabilidad social en las universidades españolas", en Actas de Jornadas de responsabilidad social universitaria organizadas por la UVA, celebradas en Valladolid los días 28 y 29 de abril de 2009, pp. 15-16.

19 DE LA CUESTA, M., ibíd., p. 14. 
a) La participación: contenidos docentes sobre RS y aprendizaje-servicio, procesos centrados en el estudiante, procesos de evaluación docente, utilización de estrategias docentes participativas tales como debates, juegos de rol, etc.

b) El medio ambiente: contenidos de desarrollo sostenible en programas, actividades de formación ambiental, estrategias docentes ecológicas, incorporación del entorno y los ecosistemas cercanos al proceso formativo, mayor utilización de recursos bibliográficos virtuales.

c) La inclusión social: formación en derechos sociales, aplicación de sistemas que faciliten los estudios en situaciones especiales, facilidad de acceso a materiales y recursos docentes para todos los estudiantes.

d) El acceso a las redes: formación de emprendedores, formación en valores cívicos, aplicación de estrategias docentes de trabajo de cooperación, oportunidades de movilidad estudiantil, actividades docentes en interacción con entes externos.

e) La toma de decisiones: formación de competencias para la toma de decisiones eficiente, fomento de la creatividad y la innovación, utilización de estrategias docentes que fomenten el trabajo en equipo.

Respecto a la promoción de la investigación, además de la básica, orientada al desarrollo del conocimiento, no puede eludirse la investigación de la Universidad desarrollada a través de convenios con las administraciones locales desde diversos departamentos de las diversas carreras, a desarrollar desde enfoques inter-disciplinares aplicados con las localidades de las que se forma parte (sin perder la perspectiva global ni caer en los localismos). Así, investigadores y docentes se encontrarían en el mismo lugar, trabajando sobre la misma problemática, desde sus especialidades respectivas, creando una sinergia de conocimientos y la inter-disciplinariedad, que pasaría a ser real y no sólo algo necesario e imprescindible que raramente se constata en la realidad.

Podemos citar aquí como indicadores de responsabilidad social:

a) La participación: estudios relativos a participación ciudadana, evaluación participativa de la $\mathrm{I}+\mathrm{D}+\mathrm{i}$ realizada, investigaciones sobre recursos-necesidades o iniciativas de la comunidad local, incorporación de estudiantes a proyectos de investigación de promoción de RS.

b) El medio ambiente: desarrollo de estudios relativos a la protección del medio ambiente, aplicación de criterios éticos para investigaciones con seres humanos, animales y ecosistemas, promoción de investigación-acción, docente y operativa.

c) La inclusión social: estudios e investigación acerca de la inclusión social a nivel local y comunitario, difusión abierta y retroalimentación de resultados de los estudios e investigaciones, programas de iniciación a la investigación para académicos jóvenes en temas sobre responsabilidad.

d) El acceso a las redes: estudios sobre recursos sociales, yacimientos de empleo... En el ámbito local, desarrollo de la investigación en conjunto con instituciones públicas y privadas, participación activa en redes nacionales e internacionales de investigación.

e) La toma de decisiones: investigación acerca de los procesos de autodeterminación ciudadana, facilidades para gestionar los proyectos de investigación con repercusión y beneficio social.

Una cuarta e importante estrategia tiene que ver con la proyección social. Ello pasa por la importancia de la investigación aplicada en la realidad social de la que forma parte la universidad con las demandas y necesidades sociales y con las posibilidades de promoción local existentes. 
La relación de la universidad con el contexto social se puede favorecer a través de los siguientes indicadores:

a) La participación: integración a redes sociales, apoyo y trabajo conjunto con organismos del tercer sector, empresas y administración pública, apertura de los espacios, procesos e infraestructura universitaria para toda la comunidad.

b) El medio ambiente: apoyo a las actividades de protección medioambiental realizadas por las redes sociales, actividades orientadas a la promoción medioambiental del contexto local y comunitario, compromiso con el entorno social y medioambiental.

c) La inclusión social: desarrollo de actividades solidarias, fortalecimiento de capacidades y competencias básicas de los sectores en situaciones especiales.

d) El acceso a las redes: convenios con empresas para prácticas profesionales, alianzas con asociaciones y colegios profesionales, realización de debates, charlas, coloquios y seminarios abiertos a la comunidad local.

e) La toma de decisiones: contribución al fortalecimiento de capacidades de la comunidad local, difusión de valores y principios institucionales hacia la comunidad, programas de apoyo al desarrollo emprendedor a nivel local.

\section{CONCLUSIONES}

A través del escenario presentado precedentemente sobre la responsabilidad social universitaria, emerge que el resultado final dependerá fundamentalmente de los agentes y de los procesos que hagan posible la voluntad de implicación en los planteamientos necesarios. Cuando nos referimos a los agentes, incluimos autoridades y personal de administración, particularmente implicados en la democracia y sostenibilidad de la organización; los docentes, investigadores y estudiantes, promotores de conocimientos, habilidades y actitudes vinculados a la responsabilidad social; sector público y privado, sociedad civil, los grupos sociales y los medios de comunicación del entorno social, que, entre sí y con la propia universidad, aúnan esfuerzos para una participación conjunta en intereses del bienestar social común más allá de los intereses particulares. Los procesos que favorecen la responsabilidad social de la universidad pasan por la promoción de capacidades humanas y culturales, el desarrollo económico, técnico-científico, ciudadano y democrático, todo ello para una calidad de vida con rostro humano.

\section{BIBLIOGRAFÍA}

BARRANCO, C.; RED VEGA, N. (2008): "Responsabilidad social organizacional y trabajo social”, Ponencia presentada en el Congreso Internacional "Ciencias, tecnologías y culturas. Diálogo entre las disciplinas del conocimiento", celebrado en la Universidad de Santiago del 30 de octubre al 2 de noviembre de 2008 (en prensa).

BOURDIEU, P. (1986): “The forms of capital”, en J. G. Richardson (ed.), Handbook of Theory and Research in Sociolgy of Education, New York: Greenword, 241-258.

COLEMAN, J.S. (1988): “Social Capital in the creation of human capital”, American Journal of Sociology, 94 (special supplement), 95-120.

CORTINA, A. (2005): "Ética de la empresa: no sólo responsabilidad social", en Ética de la empresa: hacia un nuevo orden global, http://www.etnor.org/html/pdf/XIV-SP-04-05.pdf (tomado el 20 de julio de 2008).

DE LA CUESTA, M. (2009): "Modelo de responsabilidad social en las sociedades españolas", en actas de Jornadas sobre Responsabilidad social universitaria, organizadas por la Universidad de Valladolid, 28 y 29 de abril de 2009. 
EQUIPO DE RECTORES (2005): Declaración sobre funciones cívicas y responsabilidad social de la educación superior, Talloires, Francia.

FUKUYAMA, E. (1995): "Social capital and the global economy", Foreign Affaire, 74 (5), septiembre, 89-103.

GARCÍA ROCA, J. (2004): Politicas y programas de participación social, Madrid: Síntesis.

GERRERA SOTILLO, A. (2005): "Sobre responsabilidad social empresarial”, www.analitica.com/va/sociedad/artículo/5900497.asp (tomado el 17 de agosto de 2009).

KLIKSBERG, B. (2000): Capital social y cultura, claves olvidadas del desarrollo, Instituto Interamericano para el desarrollo social, 7, 1-37.

KLIKSBERG, B.; TOMASSINI, L. (2000): Capital Social y Cultura: Claves estratégicas para el desarrollo, Banco Interamericano de Desarrollo (BID), Editorial Fondo de Cultura Económica

KLIKSBERG, B. (2005): Más ética, más desarrollo, Buenos Aires: Temas, 5. a ed.

MARTÍNEZ, R. (1995): "Redes sociales. Más allá del individualismo y del comunitarismo», en E. Dabas y D. Najamanovich (comp.), Redes. Lenguaje de los vínculos: hacia la reconstrucción y el fortalecimiento de la sociedad civil, Buenos Aires: Paidós.

MATURANA, H. (2004): “Objetividad, responsabilidad y ética”, http://www.periodismo.uchile.cl/noticias/2004/anoacademicoinaug.html (tomado el 25 de abril de 2006).

MORIN, E. (1996): "El pensamiento ecologizado", http://www.ugr.es/ pwlac/G12_01Edgar_Morin.html (tomado el 25 de abril de 2008).

MORIN, E. (2001): Los siete saberes necesarios para la educación del futuro, Barcelona: Paidós. Puede verse también sobre este tema y de este mismo autor, http://www.globalizacion.org/ciudadania/MorinFronteraPolitico.htm.

MORIN, E. (2007): "Fronteras de lo político", http://www.globalizacion.org/ciudadania/MorinFronteraPolitico.htm (tomado el 25 de abril de 2008).

NAVARRO LÓPEZ, V. (2003): “Crítica del concepto de capital social”, en Sistema. Revista de Ciencias Sociales, 172, 27-36.

OEA (Organización de Estados Americanos) - BID (Banco Internacional de Desarrollo) (2007): “¿Cómo enseñar ética, capital social y desarrollo humano en la Universidad?”, Módulo 2. Responsabilidad social Universitaria: ética desde la organización, http://www.educoas.org/portal/ineam/cursos_2007/ ETICA-E102_07.aspx?culture=es\&navid=241 (tomado en agosto de 2009).

PUTNAM, R.D. (1993): "The prosperous community. Social capital and public life", The American Prospect, 13.

RODRÍGUEZ FERNÁNDEZ, J.M. (2009): "Responsabilidad social universitaria. Desafíos ante un cambio de época", en Actas de las Jornadas sobre Responsabilidad social en la Universidad, organizadas por la Universidad de Valladolid, 28 y 29 de abril de 2009.

UNESCO: “Conferencia Mundial sobre Educación Superior”, Debate Temático sobre la Responsabilidad Social, la autonomía y la libertad académica de las universidades.

VALLAEYS, F. (2004): en A. M. Díez, La responsabilidad de la universidad en la promoción del capital social, Biblioteca virtual de Derecho, Economía y Ciencias Sociales, http://www.eumed.net/libros/ 2008b/402/Responsabilidad\%20Social\%20de\%201as\%20Universidades (tomado el 08/09/2009).

\section{DIRECCIONES ELECTRÓNICAS}

http://www.iadb.org/ética (tomado el 20 de agosto de 2009).

http://www.clarin.com/suplementos/libros/2006/03/10/masetica.pdf (tomado el 20/08/2009).

http://www.complexus.org/garcia/esppresapc.htm (tomado el 10 de agosto de 2009).

http://www.eumed.net/libros/2008b/402/Responsabilidad\%20Social\%20de\%20las\%20Universidades. htm (tomado el 12 agosto 2009).

http://palestra.pucp.educ.pe/index.php?id=111 (tomado el 10 de agosto de 2009). 